\title{
Evaluating Strategies To Collect Micrometeorites From Rainwater For Citizen Scientists
}

\author{
Mel Blake, University of North Alabama, USA \\ James McKee, University of North Alabama, USA \\ Richard Statom, University of North Alabama, USA \\ Chiong Qiu, University of New Haven, USA \\ Francis Menapace, University of North Alabama, USA
}

\begin{abstract}
Micrometeorites originate from small pieces of rock from space colliding with the Earth's atmosphere at high velocity, such as the Perseid meteors which hit the atmosphere at $60 \mathrm{~km} / \mathrm{s}$. When they do so, they burn up, causing a flash of light that we see as a meteor. Many groups have been successful collecting these particles using various devices. Such activities make great science projects for middle and high school students, and we plan to start a program to train students in the collecting methods and get them interested in science, technology, engineering and math (STEM) careers. Various methods are used to collect micrometeorites from rainwater, but little work has been done to assess the most efficient method of collecting these particles from space and then analyzing them. Before we began our citizen science project, we determined that it was necessary to conduct a pilot project to determine the most effective method of collecting micrometeorites from rainwater. Four collecting methods were tried and the method that collected the most micrometeorites was also the simplest, that being a simple bucket under the downspout of the gutter system of a house and a magnet which is then run through the bucket to gather the meteorites.
\end{abstract}

Keywords: Micrometeorite Collecting; STEM Outreach

\section{INTRODUCTION}

etting more students interested in STEM careers is a national priority (U.S. Department of Education, 2016), and many resources are being utilized to this end. One key aspect is to introduce the excitement of science by getting students to do scientific investigations and experience it themselves. So-called citizen science projects have emerged in nearly every field and are often built off the synergy between enthusiastic amateurs and the professional community. We desire to conduct a program of getting students interested in science by collecting micrometeorites from rainwater, which provides a compelling topic to get students interested in STEM careers. Project-Based Learning (PBL), or learning-by-doing, involves having students depart from the traditional classroom approach and instead having them conduct open-ended investigations in order to learn. Various studies have shown that this approach to learning is effective in achieving improved student engagement and learning. Holm (2011) reviews the literature on the effectiveness of PBL activities from K-12. The key to successful PBL is to make the content central to the curriculum goals, and to ensure that teachers guide the students to prevent false starts, and so the fact that the actives are student-centered does not mean teachers do not need to be actively involved. Holm presents a summary of results from different studies, which demonstrate that students who do PBL understand the scientific method better and improve IT skills. However, recent work shows that teachers have difficulty executing PBL, and its effectiveness is not entirely proven (Schaffhauser, 2017). A PBL activity involving collecting and studying micrometeorites would be effective only if properly designed and conducted.

About two tons of material impacts the Earth's atmosphere each day (Lunsford, 2009). A micrometeorite is created when a small meteoroid encounters the Earth's atmosphere. The intense heat melts the meteoroid and creates a flash of light that is seen as a meteor. The parent bodies of the meteoroids are typically comets, although several asteroids are also known sources, such as the Geminid meteors which come from the asteroid 3200 Phaethon (REF). The melted 
rock from the meteoroid becomes part of the dust in the air and has been collected in Antarctica and backyards. Collecting this material has been done by many people and has produced science fair projects, and gotten students interested in science (Muhs, 2004).

One key issue with regards to collecting micrometeorites from rainwater is terrestrial contamination. Anselmo (2007) has suggested the micrometeorites collected in such projects may instead be terrestrial contamination rather than celestial in origin (Genge, Larsen, Van Ginneken, \& Suttle 2017). The environments where the micrometeorites are found is suspicious, with more being found in urban areas low to the ground than in the countryside. Anselmo goes on to examine material ejected from soldering machines and lighters and shows that they bear similar appearance to the micrometeorite candidates, with some being hollow. Genge et al. (2017) note the problem of terrestrial contamination, but report that they collected 500 micrometeorites from rooftops, and examined their chemical composition, finding they are silicates with compositions consistent with meteoritic materials and the presence of rareearth elements. It can therefore be concluded that while terrestrial contamination is an issue, with false-positives probably outweighing true micrometeorites, particularly in urban environments, micrometeorites can be found on rooftops and in rainwater. Micrometeorites collected in this manner should be considered candidates until chemical and microscopic analyses can be conducted on each particle, and this is the language that we will adopt in this paper. Many researchers prefer samples from Antarctica (Thompson, 2012; Maurette 2006) due to such contamination issues.

The rocky micrometeorites are typically a few hundred microns across and can be seen under a low power (10X) microscope. Since they are essentially small samples of comets, if they can be successfully collected and separated from terrestrial samples, then they might offer insights into the parent objects. There are two major types of meteorites based on their chemical composition: rock-like and metal-like. The rocky meteorites are mostly made of inorganic crystals and the metallic ones are mostly iron-nickel alloy. Typically, inorganic crystals and metal alloys can withstand higher temperatures without evaporation than ice and organic materials. Detailed chemical analysis might help assess if a particle is of non-terrestrial origin (Genge et al. 2017). In addition, since micrometeorites must descend from the high atmosphere, studies of the number of observed meteors versus the number of micrometeorites collected might help studies of the upper atmosphere.

We plan to develop a program for high school students to participate and collect such micrometeorites. These students will attend a workshop to learn about micrometeorites and how to collect samples. They will then be given sample containers and gather micrometeorites at their schools or homes. After a semester of collecting, they will return to examine their samples and obtain SEM scans of some of their micrometeorite candidates. Data on the location, rainfall and when the micrometeorites were found, and the SEM scans will be added to a database that will be used to study the fall of these objects over time and in different environments. Such data will make good project for science fairs and studies of the long-term number of micrometeorites that call can be studied, as well as whether events like a Leonid meteor storm increases the number of micrometeorites collected, and if so, how long does it take for the effects to be noticed, and for how long. Meteor cameras could also be incorporated into the project to count meteors falling in the area micrometeorites are collected to study any connection with those statistics.

Students who do a project related to micrometeorites should benefit in several ways. First of all, they will learn about the solar system, and the importance of studying primitive objects in solar system in order to gain a better understanding of the formation of the solar system. In addition, they will learn about the origin of meteors and meteorites and how we can obtain samples for comets and asteroids through the collision of the Earth with the micrometeoroid particles. The identification of the micrometeoroid candidates chemically (see below) will also inform students about the chemistry of the micrometeoroid particles, and how to verify the validity of a sample. Through conducting studies of the samples once identified, students should gain an improved understanding of the scientific method (Holm, 2011), and statistical methods for analyzing the data. If presented at science fairs, the students should gain experience in public presentations and responding to questions about their projects and get some sense of accomplishment that may motivate them to pursue STEM careers. 
Before such projects can be conducted, what is needed are systemic collecting efforts. This is an ideal project for middle and high school students if effective methods of collecting candidate micrometeorites can be identified. Collection methods vary from collecting samples from downspouts with magnets (Miller, 2013) to using a magnet in a bucket left outside to collect rainwater (Spangler, 2016). Similarly, Larsen (2016) encourages citizen science studies of micrometeorites with Project Stardust. Here we describe our efforts to examine several collection methods in order to determine the best choice for students to gather samples. Our motivation here is that there does not seem to be much published literature examining the best way to collect micrometeorites, and we hope to remedy this with this and future efforts. We also present some preliminary examination of a micrometeorite collected in our project.

\section{COLLECTION METHODS}

Various methods of collecting micrometeorites have been discussed in the literature. One method involves using a plastic sheet left outside for a week or two under which a magnet is run to gather samples (Miller, 2013). Another method is to place a bowl or bucket under a downspout (Hamilton, 2007). With this method the heavier particles settle to the bottom of the bucket and a magnet is used to gather the samples. Other methods involve attaching magnets in a down-spout directly to gather samples of micrometeorites.

The primary aim of this pilot study was to examine several methods of collecting samples to determine which ones produced the most candidates. The first two methods we tested involved connecting the collectors directly to the down-spout of a house; these have the advantage of using the entire roof of the house to gather samples increasing the volume of water and therefore potentially increasing yield. These collectors were one of two types. The first was a simple bucket placed under the downspout of a house to collect rainwater (Figure 1a), with the meteorites settling to the bottom to be collected later. A screen was placed over the bucket to prevent sticks and larger material from falling in the bucket. A second procedure involved connecting a P-trap to the downspout with magnets attached to the bottom of the P-trap (Figure 1b). As water streams past the magnets, the meteorites get collected because of the iron and nickel which they contain. A modification of these collection methods was to place two control traps away from the down-spout identical to the first two Figure $1 \mathrm{c}$ and $\mathrm{D}$ ). Due to lower volumes of water, we expected the traps that were not connected to the downspout to collect less material, but they provided independent assessment of the two types of trap. Figure 2 illustrates each of these traps. 
Figure 1. (A) Top left. A simple bucket and screen under a downspout. (B) Top right. A P-trap and magnet collector on a downspout. (C) Bottom left. A P-trap with a funnel held down by cinder blocks. (D). A simple bucket and screen arrangement. Neither trap C or D were connected to a downspout.
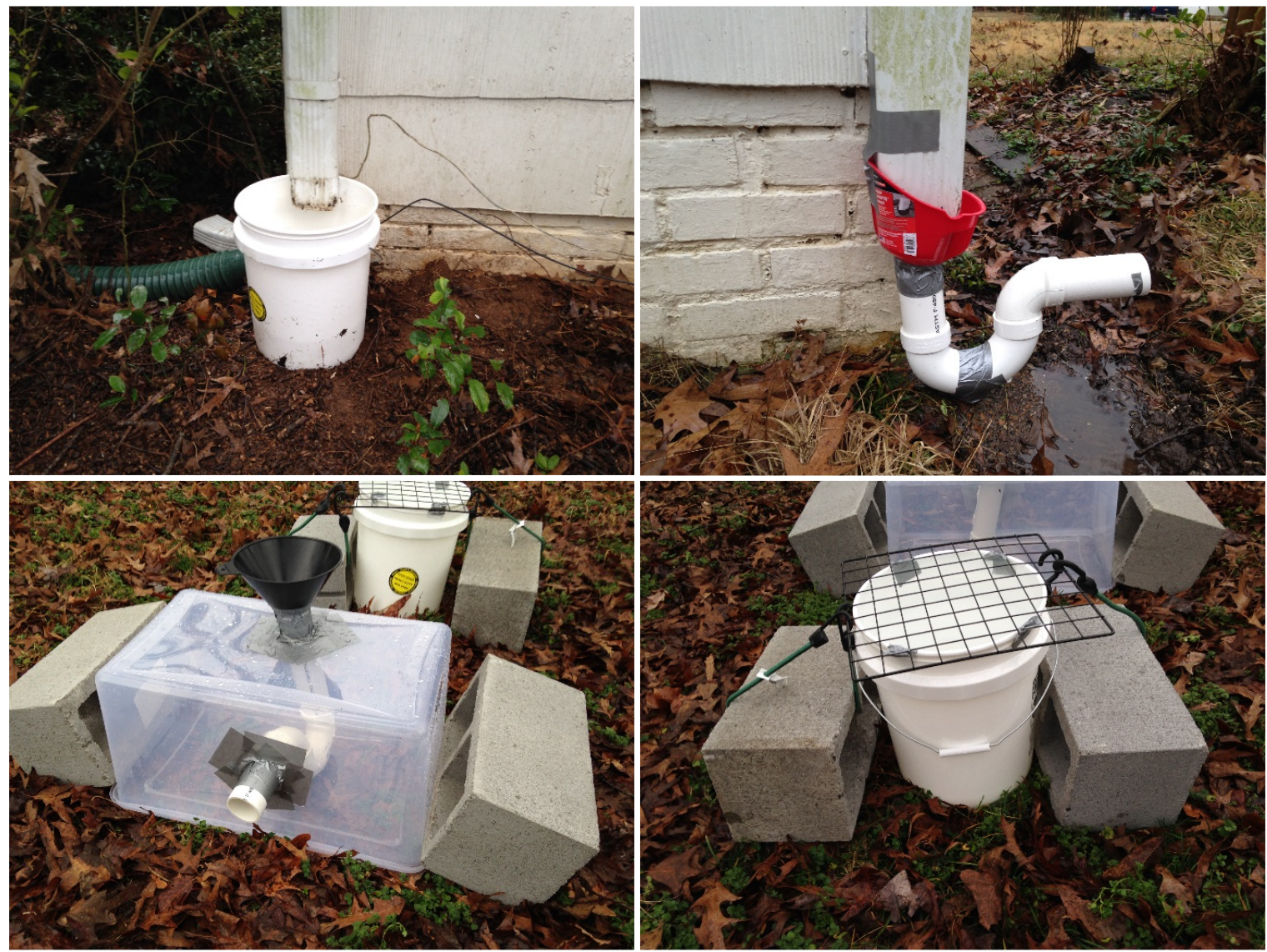

Photo credit: J. McKee 
Figure 2. Illustrations of the four types of traps that were tested. Top left we show the downspout bucket trap, top right is the downspout P-trap, bottom left is the open air P-trap, and bottom right is the open-air bucket.

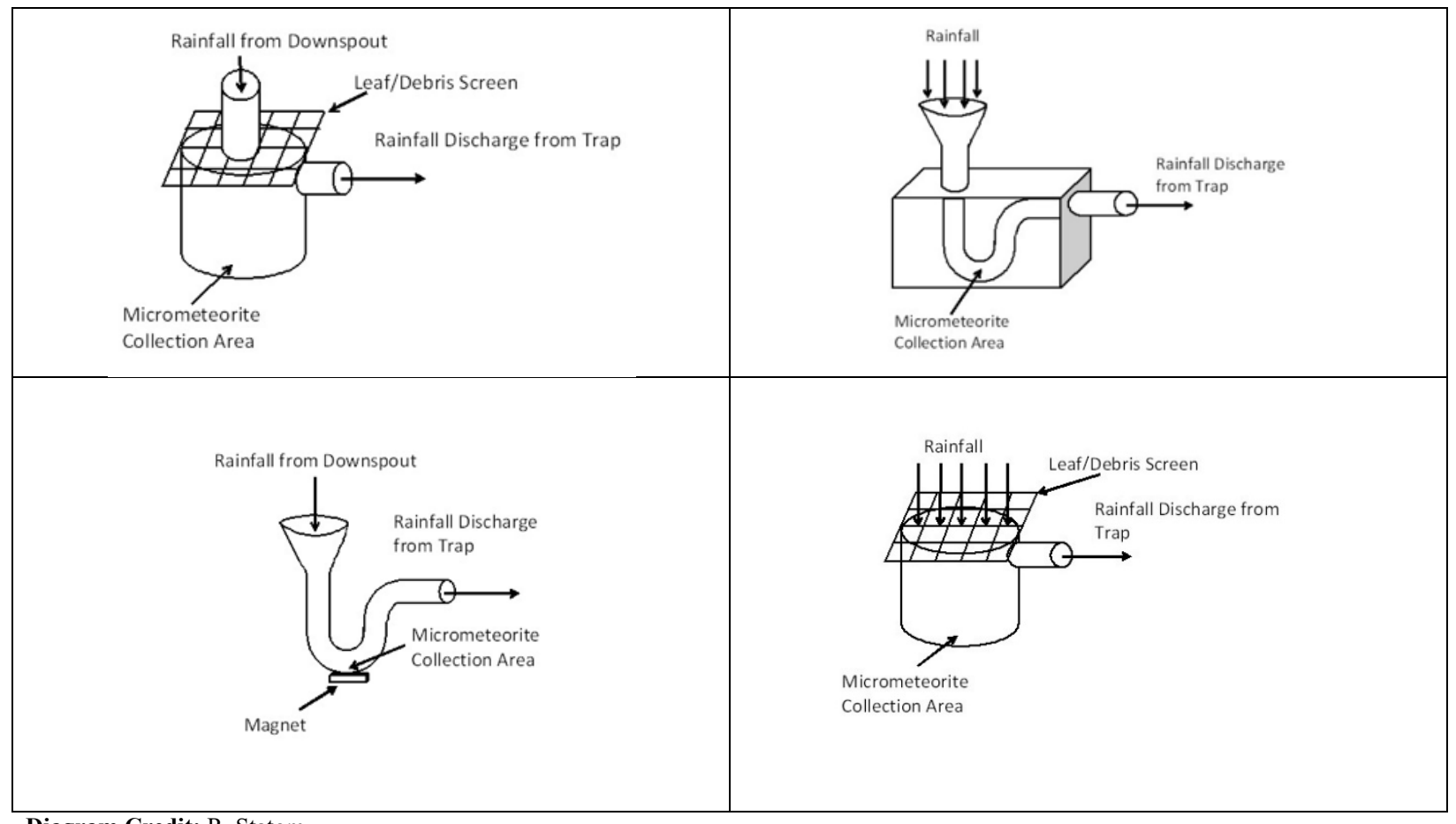

Diagram Credit: R. Statom.

The traps were placed out to collect samples material for about one week and the samples were then collected, and candidate micrometeorites were then identified. Table 1 gives the date and amount of rainfall for each collecting period. Rainfall data was taken from the Weather Underground data (Weather Underground 2016). Rainfall was relatively low during this time, and so we might expect more micrometeorites if rainfall were higher, something we plan to test in the future. For the P-traps, the magnets were removed, and water rinsed through the traps with a funnel and filter paper catching the material. These were then dried, and a microscope used to identify micrometeorites by their round appearance. For the bucket traps, the micrometeorites were collected from water that accumulated in the buckets along with various debris and the potential micrometeorites. The first run we did, the buckets gave a tremendous amount of sticks, dead insects and other debris that took hours to sort through. We then decided to use what we termed "magnetic fishing" to collect meteorites. A strong magnetic was placed in a plastic bag and the water in the bucket was stirred up and a magnet was then run through the water, capturing micrometeorites which were then placed in sample jars. A 10-20X dissecting microscope was used to identify the micrometeorites for both types of trap. Candidates were then examined under a $40 \mathrm{X}$ high power microscope for final identification.

Table 1. Rainfall data for Florence Alabama during collecting periods.

\begin{tabular}{c|l|c}
\hline Collecting Period & \multicolumn{1}{|c}{ Dates } & Precipitation (in) \\
\hline 1 & February 23-March 2 & 0.68 \\
\hline 2 & March 7 - March 15 & 0.90 \\
\hline 3 & April 5 - 12 & 1.63 \\
\hline 4 & April 13 - 19 & 0.83 \\
\hline 5 & April 20 - 26 & 0.01 \\
\hline 6 & April 27 - May 3 & 1.22 \\
\hline 7 & May 4 - May 12 & 1.03 \\
\hline 8 & May 13 - 19 & 0.08 \\
\hline Total & & 6.38 \\
\hline
\end{tabular}




\begin{abstract}
ANALYSIS
Table 2 summarizes the results of the experiment, giving the total, cumulative number of micrometeorite candidates collected in each trap for the eight weeks of collecting. Here we have simply added up the total number each trap collected in all, and so the data in Table 2 represent cumulative results for each trap over eight weeks. Since the four traps were all placed in the same location and therefore were subjected to the same rainfall conditions, we do not scale or weight the number of meteorites per trap per rainfall, since such weighting would be same for all the traps. We determined which traps were most effective by examining the number of micrometeorites collected by each. The numbers of micrometeorites were expected to be small. We can attempt to estimate the number of micrometeorites expected to be collected based upon the rainfall. There was a total of 6.38 inches of rain in total that fell during the experiment. According to the U.S. Geological Survey rainfall calculator, (USGS 2018), a one foot by one foot area of land will receive 1 gallon of water if one inch of rain falls. This would translate into 6.38 gallons of water per square foot over the time we collected micrometeorites, which is 73.91 gallons per square meter (cumulative), or 279.77 liters per square meter. The funnel on the P-trap collector had a diameter of $18.5 \mathrm{~cm}$, giving an area of 0.0269 $\mathrm{m}^{2}$. The open-air bucket had a diameter of $30 \mathrm{~cm}$, translating into an area of $0.071 \mathrm{~m}^{2}$. The estimate of the collecting area for the downspout traps is problematic. The water that hits the roof pours into the downspout, with presumably the water hitting the roof on the side of the downspout supplying it with water, and if the traps are on either side of the roof, equal volumes of water would go through each trap. With this assumption, the roof in question is $125.6 \mathrm{~m}^{2}$. One half of the water poured down one side of the roof, with a downspout on each side, each getting half the water. So we have an effective area for each trap of one quarter the area of the roof, or $31.4 \mathrm{~m}^{2}$. This results in an estimated volume of water of $6.38 \mathrm{in}$.
\end{abstract}

Larsen (2018) describes the number of micrometeorites per year for a roof of about $100 \mathrm{~m}^{2}$. He states that one $0.1 \mathrm{~mm}$ object is expected to fall per square meter per year, but an average micrometeorite is $0.3 \mathrm{~mm}$, and we expect about four of these per year on a $100 \mathrm{~m}^{2}$ roof, but with larger $0.4 \mathrm{~mm}$ objects also falling. He therefore estimates one or two new micrometeorites per year on a $100 \mathrm{~m}^{2}$ area, independent of the amount of rain or snow, but with rainfall affecting the actual numbers. It is therefore difficult to predict the estimate for the number of micrometeorites we might expect in out traps. It is clear that a greater collecting area will collect more candidates, and greater rainfall will affect the results in ways difficult to quantify. If the rainfall is the same for all traps, then presumably we expect a greater collecting area to result in a greater volume and a larger number of candidates. It therefore is not surprising more candidates were obtained from the downspout traps which collect from much great effective collecting areas. Given the numbers it is surprising as many micrometeorite candidates were detected as there were. We expected few if any candidates from the smaller traps at all over an eight week period, and perhaps one or two in the larger traps. This suggests significant contamination in these traps, and indeed particles from the roof tiles were found in the samples in addition to what we concluded were micrometeorites.

We immediately rejected the open air P-trap arrangement largely because of there being so much other material in the water that it was impossible to do effective collecting, resulting in just one micrometeorite candidate being collected from that trap. Likewise, the open-air bucket trap had a problem that even with a funnel to try to increase the amount of water entering the trap, no micrometeorites were collected in this method. The volume of water from the downspout was expected to be greater because all water falling on the roof of the house would be sampled. The P-trap under the downspout collected three micrometeorite candidates while the simple bucket collected six micrometeorite candidates. We conclude that the simplest collection method also seems to be the most effective method for collecting micrometeorites. 
Table 2. Results of Candidate Micrometeorite Collections for Micrometeorite Traps

\begin{tabular}{c|c|c|c|c}
\hline Method & Bucket + Downspout & P-trap +Downspout & Open-air Bucket & Open Air P-trap \\
\hline Number Collected & 6 & 3 & 0 & 1 \\
\hline
\end{tabular}

Table 3. The Expected number of Micrometeorite candidates for each trap

\begin{tabular}{l|c|c|c}
\hline \multicolumn{1}{|c|}{ Trap } & Collecting Area $\left.\mathbf{( m}^{\mathbf{2}}\right)$ & Water Volume (L) & $\begin{array}{c}\text { Expected Number of } \\
\text { Micrometeorite Candidates }\end{array}$ \\
\hline Open air P-trap & 0.0269 & 7.5 & $<1$ \\
\hline Open Air Bucket & 0.071 & 19.9 & $<1$ \\
\hline Down-Spout P-Trap & 31.4 & 8785 & $1-2$ \\
\hline Down-Spout Bucket & 31.4 & 8785 & $1-2$ \\
\hline
\end{tabular}

\section{FINDINGS}

As noted above, the primary aim of the project was to determine the most effective methods for micrometeorite collection so that it might be replicated for a citizen science project involving high school students. The down spout bucket collected twice as many micrometeorites as the P-trap under the same circumstances and therefore we suggest it is the most effected method for collection. A secondary part of the project was to attempt to assess the crystallography and chemical composition of the micrometeorites. This proved less successful. Figure 2 shows a photo of a micrometeorite collected in this project. It is a prototype specimen with a smooth, rounded structure and small pits or holes where gases would have escaped as the particle melted while burning up in the atmosphere. Unfortunately, it is also opaque and crystallography requires the material to transmit light so polarization of light can be measured. The samples collected were therefore not able to be studied for their crystal structure. Future samples may not be opaque, so we still hope to conduct these types of studies.

The study of the chemical composition of the micrometeorites was also not possible for a separate reason. We obtained a scanning electron microscope (SEM) image (Figure 4) of the same micrometeorite that is shown in Figure 3. The image gives great detail and shows a region on the micrometeorite with a differing structure than the rest. Unfortunately, the object is only 400 microns across. Assuming a density value of $8 \mathrm{~g} / \mathrm{cm}^{3}$, the observed micrometeorite only has a mass of about 0.3 micrograms. It therefore does not have enough mass to be effectively measured with the equipment available, and we would need to combine many particles in one sample to get enough for a measurement, which would mean we would get an average of many particles. Several chemical analysis approaches may be used for the iron and nickel content analysis once enough micrometeorites are collected, including Mass Spectrometer, the Ultraviolet-Visible Spectrophotometer (UV-VIS), SEM and Atomic Absorption Spectrometer (AAS). A more specialized and more sensitive mass spectrometer designed specifically for single particle chemical composition detection would need to the obtained to do such work on one particle. However, the impressive SEM image suggests that SEM studies might be productive in lieu of chemical data and crystallography, providing information about the surface of the micrometeorites without damaging the bulk part. We are seeking funds for attachments for the SEM that can do surface compositions that would greatly improve the analysis of the micrometeorites. The micrometeorites will then be dissolved in strong acid solutions that convert the iron and nickel into ions in the solutions. Direct analysis using AAS can then be performed. Alternatively, appropriate chemical reactions can be used to convert iron and nickel ions into complexes with distinctive colors for UV-VIS analysis. For example, iron ion can react with phenanthroline to form a red complex for concentration analysis. 
Figure 3. This is a photograph under high power microscope of a micrometeorite collected in this project. It has the characteristic round shape and pitting on its surface.

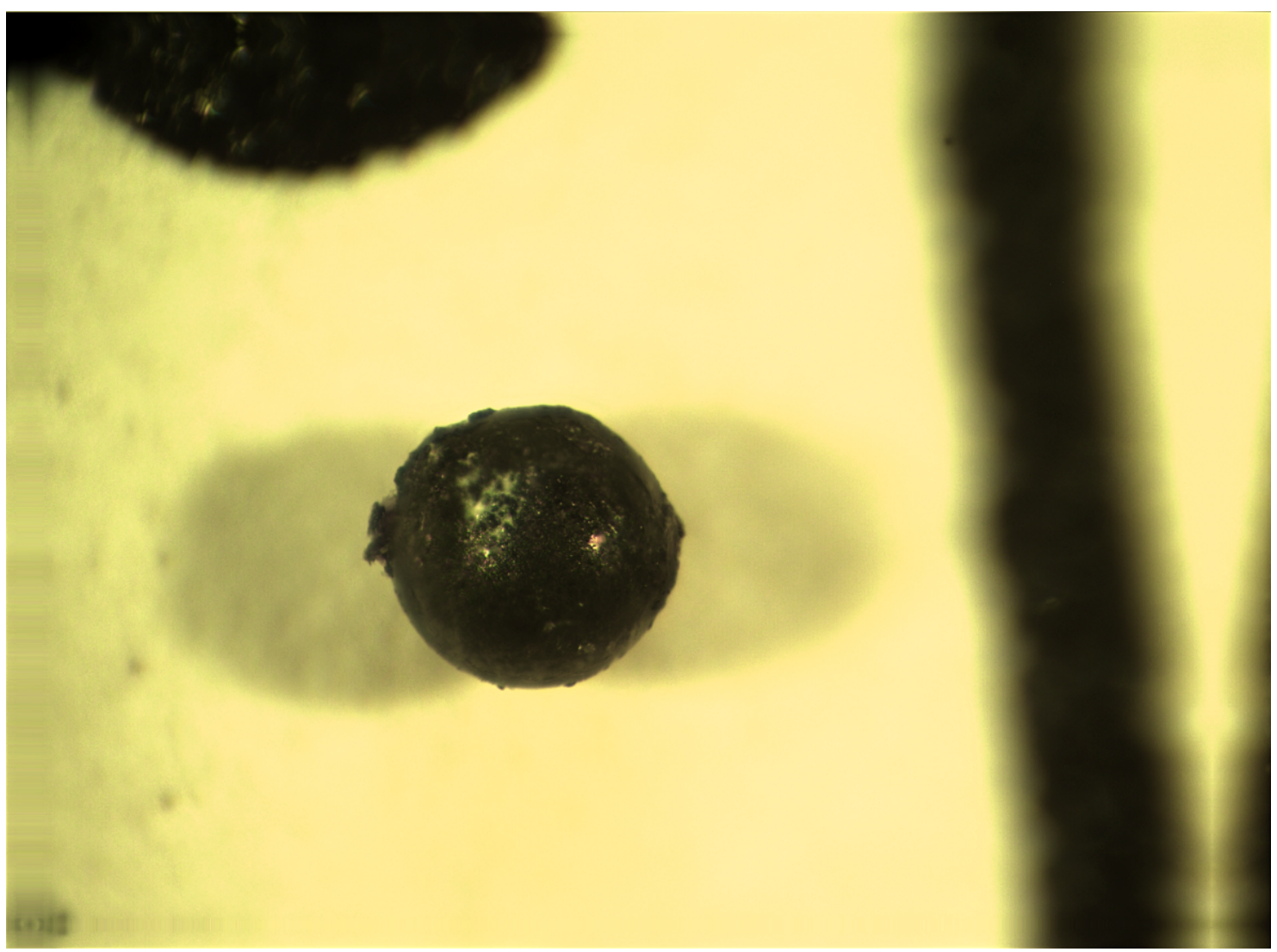

Photo credit: J. McKee.

\section{Future Work}

Now that we have identified a good way to collect micrometeorites from rain water, these results can be used to create collaborative programs for school students to gather micrometeorite candidates and partner with universities and colleges to analyze samples. It is clear that contamination from terrestrial sources is a major factor in using the samples to study micrometeorites. While the candidates can be identified from their appearance as small round particles in the microscope, without a chemical analysis, they cannot be verified as true micrometeorites. A one possible project that could be done would be for school students to visit a host university or college and learn about the micrometeorites, how to collect them and then to return to their schools and collect samples for several weeks or months, storing the samples. They could then return to the university, which would then analyze the samples to identify the true micrometeorites. A database could then be set up with the date, time and location of each verified sample that would be available to participants, and possibly to the general public to use for studies. One other possible model for a collaborative project would be for the students to visit, but instead of returning with their samples, the university or college would receive the samples in the mails from the school students and analyze them, and then return the results to the students. One additional benefit to this is that undergraduate students could be trained in how to do the chemical analysis and therefore gain valuable lab experience in chemical analysis. 
Figure 4. A SEM image of the micrometeorite shown in Figure 3. The micrometeorite is only about 400 microns across. The pitting of its surface is apparent as well as a region to the right with differing characteristics.

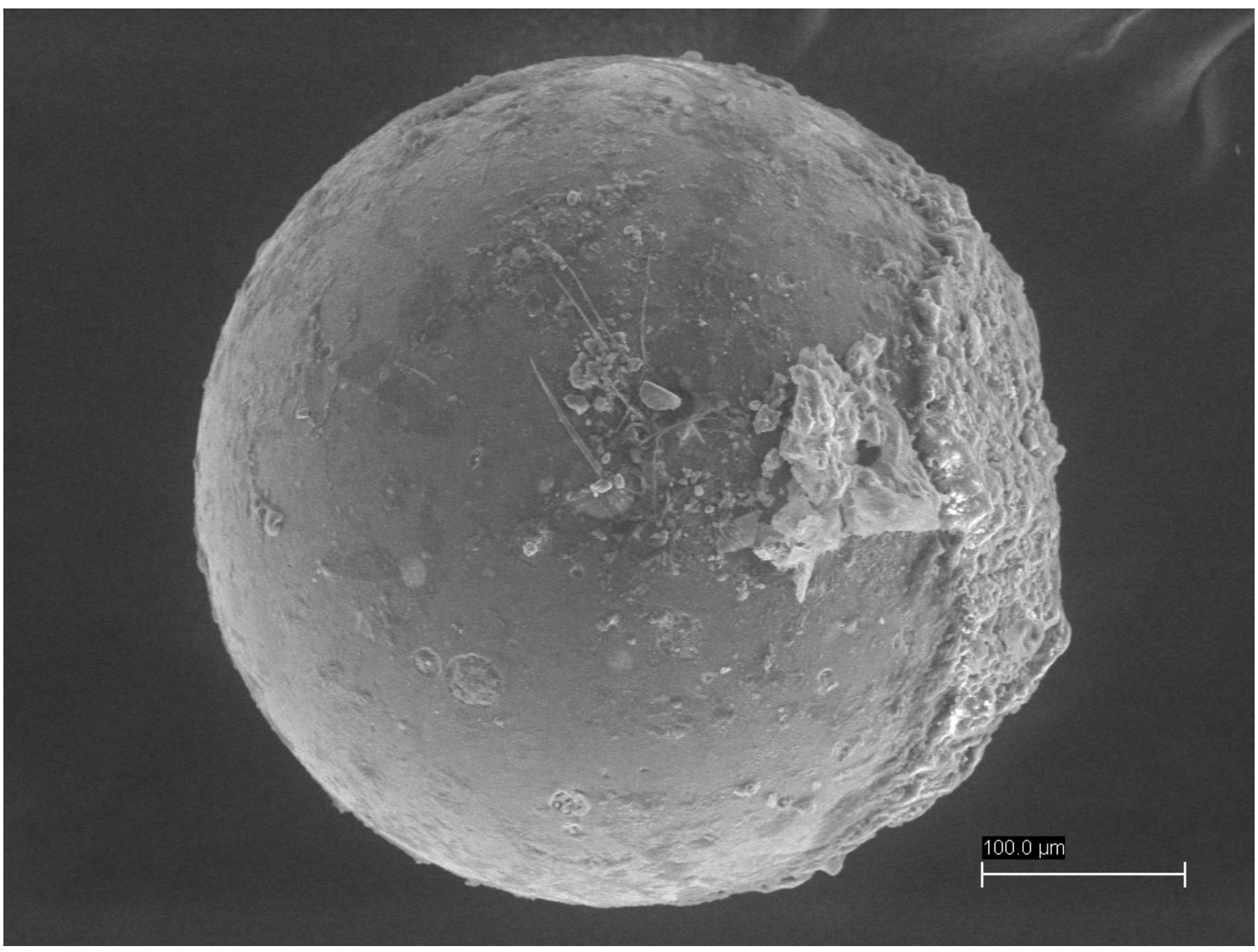

Photo Credit: F. Manapace

Once the database of confirmed micrometeorites is established, several useful investigations could be conducted. One useful project would be to better understand the significance of the contamination problem its self. Collecting samples of a wide geographic area, in rural as well as urban areas could help show where contamination is worse, and even factor in things like prevailing winds coming from the direction of large industries and potential seasonal effects due to snow and ice storms. Since the date of the collections would be known, studies could be done as the whether the number of micrometeorites are tied to the recorded number of meteors in an area. A parallel project to image and count meteors in an area could be done to see if any correlations exist. Inexpensive meteor cameras are now available for such studies. If that were not possible, then records from the Meteoritic Society could be used instead. Another possible use of the data, since a chemical study would be available for each meteorite would be statistical studies of the makeup of the meteorites and where the difference meteorites might have originated in the solar system. Some meteors originate from asteroids instead of comets, and we might expect the chemistry to be different from the two sources.

In summary, we have studied a simple method for collecting micrometeorite candidates from rainwater that can be reproduced inexpensively by school students. If collaborations can be formed between such students and local colleges then interesting studies can be made with such samples suitable for science fair projects and for generating interest in STEM careers among participating students. 


\section{ACKNOWLEDGEMENTS}

We recognize the support of the UNA Faculty of Arts and Science through a Student-Faculty research grant which helped fund this project. We also thank Dr. George Makowski for allowing us the use of his home and property for the collection of the micrometeorites, and for Dr. Larsen's correspondence about micrometeorite statistics. We gratefully acknowledge the comments and suggestions of the anonymous referees which greatly improved this paper.

\section{AUTHOR BIOGRAPHIES}

Mel Blake is an astronomer who works on binary and variable stars and also operates the UNA Planetarium and Observatory. He recently has become interested in B(e) stars in different environments and their variability with time.

James McKee is an undergraduate student in Physics who is interested in all things space and astronomy related.

Francis Menapace is a Botanist who works with plant micromorphology (SEM). He is also interested in the vascular plant flora of Northwest Alabama.

Chong Qiu is an analytical chemist who works in the field of environmental chemistry, especially issues related to the role of amines in the atmospheric processes and the transformation of ambient soot.

Richard Statom's research focuses on hydrology, hydrogeology and water resources. He teaches Earth Science, Physical Geology, Mineralogy, Meteorology, Hydrogeology, Technology and the Environment.

\section{REFERENCES}

Anselmo, A. (2007). Observation of false spherical micrometeorites. Retrieved from https://arxiv.org/ftp/arxiv/papers/0708/0708.4276.pdf

Genge, M. J., Larsen, J., Van Ginneken, M., \& Suttle, M. (2017). An Urban Collection of Modern-day Large Micrometeorites: Evidence for Variations in the Extraterrestrial Dust Flux Through the Quaternary, Geology, 45, 119.

Hamilton, C. J. (2007). An educators guide to micrometeorites. Jet Propulsion Laboratory. Retrieved from http://solarviews.com/eng/edu/micromet.htm

Holm, M. (2011). Project-based instruction: A review of the literature on effectiveness in prekindergarten through $12^{\text {th }}$ grade classrooms. River Academic Journal, 7, 1.

Larsen, R. (2018). Private Communication.

Larson, J. (2016). In search of star dust. Retrieved from http://www.cbc.ca/news/canada/sudbury/in-search-of-stardust-findingmicro-meteorites-on-your-roof-1.3643023

Lunsford, R. (2009). Meteors and how to observe them. Springer Verlag.

Maurette, M. (2006). Micrometeorites and the mysteries of our origins. Springer-Verlag.

Miller, R. (2013). How to collect micrometeorites in your back yard. Retrieved from http://io9.gizmodo.com/5984951/how-tocollect-meteorites-in-your-backyard

Muhs, E. (2004). Micrometeorites. Retrieved from https://icecube.wisc.edu/outreach/activity/micrometeorites

Schaffhauser, D. (2017). Project-based learning: 'Promising but not proven,' researchers say. The Journal, https://thejournal.com/articles/2017/11/08/project-based-learning-promising-but-not-proven.aspx

Spangler, S. (2016). A meteorite hit my house. Retrieved from https://www.stevespanglerscience.com/blog/2004/10/21/ameteorite-hit-my-house/

Thompson, R. (2012). The Dark Flight of Meteorites. Making the Geologic Now, ebook, edited by E. Ellsworth and J. Kruse

U.S. Department of Education (2016). STEM 2026 A Vision for Innovation in STEM Education, Report.

U.S. Geological Survey (2018). Retrieved from https://water.usgs.gov/edu/earthrain.html

Weather Underground (2016). Retrieved from https://www.wunderground.com/history/ 